\title{
Chinese oncologists' knowledge, attitudes and practice towards palliative care and end of life issues
}

\author{
Xiaoli Gu ${ }^{1}$ and Wenwu Cheng ${ }^{1,2^{*}}$
}

\begin{abstract}
Background: Oncologists` knowledge and attitudes to palliative care (PC) and end of life (EOF) should be highlighted in order to give them effective education. This study is intended to provide a descriptive analysis of oncologists' knowledge, attitudes and practice toward PC and EOF issues in Mainland China.

Methods: The questionnaire survey with 24 items investigating oncologists' demographic information, knowledge and attitudes toward PC and EOF issues was conducted among Chinese Oncology clinicians.

Results: The participants had a mean of 10.10 years practice in oncology. 43 (31.2\%) participants had received PC education. $73.9 \%$ of the participants believed that PC should be considered when patients were not suitable to take surgery, radiotherapy, chemotherapy and other anti-cancer therapies. $72.5 \%$ of the participants believed that early PC integration can improve the quality of life in patients. Most of the oncology clinicians (73.9 \%) believed that the doctor-in-charge was the appropriate person to inform patients of the diagnosis. However, only 11 participants chose to inform the diagnosis and disease prognosis to the patients, whereas $39.9 \%$ of the participants chose to disclose it to Family/Caregivers first. Besides, Chinese oncologists were obviously unfamiliar with the concepts of euthanasia and related issues.
\end{abstract}

Conclusions: This study indicated the insufficient knowledge toward PC and related issues of the Chinese oncologists. More attention should be paid on the education of PC among Oncologists in Mainland China.

Keywords: Oncologists' knowledge, Palliative care, Palliative care education, Physician education

\section{Background}

The growing aging population, the increasing incidence rates of cancer and the huge number of terminally ill cancer patients made palliative care (PC) important and imperative for cancer patients. In recent years, there has been an increase in PC services in developing countries, including Mainland China [1]. However, compared to Western countries, PC has not been recognized as an important specialty in Mainland China yet. PC experts have suggested that oncologists' knowledge and attitudes should be associated with their practices in care for patients [2]. But oncologists were reported to be under the misapprehension that they have sufficiency ability to deal

\footnotetext{
* Correspondence: wenwucheng@yahoo.com

'Department of Integrated Therapy, Fudan University Shanghai Cancer Center, \#270, Dong An Road, Shanghai, People's Republic of China ${ }^{2}$ Department of Oncology, Shanghai Medical College, Fudan University, Shanghai 200032, China
}

with patients' symptoms. Therefore, they do not tend to refer patients to PC specialists, because PC was still misconceived as doing nothing by some medical pratictioner [3]. Acutually, onocologists' insufficient understanding of PC service is still one of the barriers to PC development. Oncologists' knowledge and attitudes to PC and end of life (EOF) should be highlighted in order to give them effective education.

A previous survey of experienced Chinese urban physichowed that a significant number of these physicians agement [4]. These years witnessed the great development of PC services in China. While, there have not national population-based studies available of the knowledge and attitudes of Chinese Oncologists in PC yet. We report on a questionnaire to survey Mainland Chinese oncologists' knowledge, attitudes practice towards $\mathrm{PC}$ and EOF issues. 


\section{Methods}

\section{Questionnaire}

The study was designed as a cross-sectional, anonymous, self-administered questionnaire survey. The questionnaire was developed by researchers on the basis of literatures review [5-8]. After studying literatures, 7 PC specialists generated a total of 50 items for an item pool based on the literature reviews and discussion. To achieve validity, an expert panel (3 palliative specialists, 3 oncologists) rated the appropriateness of each item. First, experts evaluated the appropriateness of each item according to four grades. Next, the mean, minimum and maximum values were calculated, and the items with less than a mean of two and a minimum of zero were deleted. Then, the argument for the item selection was repeated including an evaluation of the way the concept was expressed. As a result, a preliminary list of 24 items was selected from the original 50 items. The final questionnaire was to assess the knowledge, attitudes and practice of the respondents covering issues related to $\mathrm{PC}$. The complete questionnaire included five categories: 1) personal characteristics such as gender, age, education, practice years and etc.; 2) concept and philosophy of PC (Q1-Q9); 3) disease information disclosure and breaking bad news (Q10-Q12); 4) end of life decision making issues (Q13-Q19); 5) euthanasia and related issues (Q20- Q24). For Q6 and Q17, respondents could choose more than one items. The research team reviewed the questions for clarity. The questionnaire was written in Chinese. It took approximately 10 to $15 \mathrm{~min}$ for experts to complete the questionnaire. A pilot study was tested in 20 oncologists for twice to assess the reliability by using Cohen's Kappa statistics, which was 0.85 .

\section{Participants and procedures}

In order to have a representative sample and generalizable results, the questionnaire was carried out in Sep 2014 to May 2015 in three hospitals and two national oncologic meetings held in Mainland China. Three hospitals included Fudan University Shanghai Cancer Center (FUSCC), $6^{\text {th }}$ people's hospital, Shanghai Jiaotong University and Kangjian Community Health Center. FUSCC is an urban, teaching, tertiary cancer center. $6^{\text {th }}$ people's hospital, Shanghai Jiaotong University is a comprehensive tertiary hospital. Survey in this hospital was conducted in Medical Oncology Department. Kangjian Community Health Center is one of the primary community hospitals. Survey in this hospital was conducted in General Medical Department. Two national oncologic meetings was the Pain Management Education meeting held in Songjiang, Shanghai and the Oncologic Nutrition Meeting held in Chongqing, Sichuan. The oncologists attending the meeting came from different hospitals all over the country in China.
The inclusion criterion for the subjects was that they were registered as Chinese oncologists including PC specialists.

The questionnaire and cover letter were distributed to participants. Among 145 oncologists surveyed, 138 respondents' answers were effective and analyzed. The overall completed response rate was $95.2 \%$. Participation in this study was confidential and anonymous, with consent taken after provision of a cover letter containing information details of the study. Consent to participate was indicated by the completion and return of the questionnaire. All answers were entered into a computerized database by investigator for confidentiality. Then data were analyzed by an independent investigator who was unrelated to the data collection. Only the researchers had access to the data.

This study was reviewed by Research Ethics Committee of Fudan University Shanghai Cancer Center. Since this study focused on professional staff only and has no involvement of patients, there is no requirement to get permit from ethics committee in China.

\section{Statistical analyses}

Statistical Package for the Social Sciences software Version 16.0 for Windows (SPSS Inc., Chicago, USA) was used for statistical analysis. Descriptive statistics (proportions, means and distributions) were used as appropriate to describe participants' characteristics and respondents. $95 \%$ confidence intervals (95\% CI) were calculated for the mean age and mean clinical practice years in Table 1.

\section{Results}

\section{General characteristics and education background}

The questionnaires of 138 respondents were analyzed. $52.2 \%$ of them were male. 84 of the participants had post-graduate education, with an average of 13.43 years of clinical experience in oncology. The participants had been in practice for a mean of 10.10 years in oncology. $42.7 \%$ of them had more than 10 years of oncological experience. 50 (36.2\%) participants were attending physicians, 25 (18.1\%) were assistant professors and 24 (17.4\%) were professors. 43 (31.2\%) participants had received PC education. The specialty of practice included Oncological surgery (5.1\%), Chemotherapy (47.1\%), Radiotherapy (4.3\%), Traditional Chinese Medicine (6.5 \%), Palliative and hospice $(27.5 \%)$. The general characteristics details of participants were in Table 1.

\section{The general knowledge and attitudes on PC}

Q1-Q5 was designed to describe oncologists' general knowledge of PC. The percentage of correct answers of Q1-Q5 was from 64.5 to $93.5 \%$. The item with 
Table 1 Characteristics of participants ( $N=138)$

\begin{tabular}{|c|c|c|c|c|c|}
\hline Variables & Number & Percent & Variables & Number & Percent \\
\hline Age (mean, $95 \% \mathrm{Cl}$ ) & 37.30 & $(35.8-38.8)$ & Gender & & \\
\hline $20-29$ & 23 & 16.3 & Male & 72 & 52.2 \\
\hline $30-39$ & 62 & 44.0 & Female & 66 & 47.8 \\
\hline $40-49$ & 40 & 28.4 & Education level & & \\
\hline$>=50$ & 13 & 9.2 & Med Bachelor & 54 & 39.1 \\
\hline Years in practice (mean, $95 \%$ Cl) & 13.43 & $(11.9-15.0)$ & Med Master & 52 & 37.7 \\
\hline $0-9$ & 57 & 41.3 & Medical Doctor & 28 & 20.3 \\
\hline $10-19$ & 41 & 29.7 & Post-doctorate & 4 & 2.9 \\
\hline$>=20$ & 40 & 29.7 & Specialty & & \\
\hline Years in cancer (mean, $95 \%$ Cl) & 10.10 & $(8.6-11.6)$ & Oncological surgery & 7 & 5.1 \\
\hline $0-9$ & 79 & 57.2 & Chemotherapy & 65 & 47.1 \\
\hline $10-19$ & 34 & 24.6 & Radiology & 6 & 4.3 \\
\hline$>=20$ & 25 & 18.1 & $\mathrm{TCM}^{\mathrm{a}}$ & 9 & 6.5 \\
\hline Title & & & Palliative/ hospice & 38 & 27.5 \\
\hline Professor & 24 & 17.4 & Others & 13 & 9.4 \\
\hline Assistant professor & 25 & 18.1 & Working Region & & \\
\hline Attending & 50 & 36.2 & Shanghai & 87 & 63.0 \\
\hline Resident & 27 & 19.6 & Non-Shanghai & 51 & 37.0 \\
\hline Fellow & 9 & 6.5 & Institutions & & \\
\hline PC education & & & $\mathrm{TCH}^{\mathrm{b}}$ & 60 & 43.5 \\
\hline Yes & 43 & 31.2 & $\mathrm{SCH}^{\mathrm{c}}$ & 14 & 10.1 \\
\hline \multirow[t]{2}{*}{ No } & 95 & 68.8 & $P C H^{d}$ & 34 & 24.6 \\
\hline & & & Cancer Center & 30 & 21.7 \\
\hline
\end{tabular}

${ }^{\mathrm{T}} \mathrm{TCM}$, Traditional Chinese Medicine

${ }^{\mathrm{b}} \mathrm{TCH}$, Tertiary Comprehensive Hospital;

' $\mathrm{SCH}$, Secondary Comprehensive Hospital;

${ }^{\mathrm{d}} \mathrm{PCH}$, Primary community hospital

the highest percentage of correct answers (93.5\%) was Q2: PC should not be provided along with anticancer treatment. 55 (39.9\%) participants had the correct answer for all five questions. For Q6, respondents could select more than one item in response. Only $32.6 \%$ of the participants agreed that referral to PC should be made when cancer was first diagnosed. $73.9 \%$ of the participants believed that PC should be considered when patients could not undertake surgery, radiotherapy, chemotherapy and other anti-cancer therapy. $29.2 \%$ of the participants would recommend PC for cancer patients when the patients attend the clinical first time. $72.5 \%$ of the respondents believed that early PC integration could improve patients' quality of life (QOL) and $54.3 \%$ of the respondents believed that PC could improve patients' survival. Details were shown in Table 2.

\section{Disease information disclosure preference}

The third part of the questionnaire dealt with questions aiming to identify participants' attitudes and practice toward disease information disclosure and breaking bad news (Q10-Q12). $44.2 \%$ participants decided to inform patients' unfavorable prognosis according to the specific situations. Although, most of the oncology clinicians (73.9 \%) believed that the doctor-in-charge was the appropriate one to inform the patient of the diagnosis. Only 11 participants chose to inform the diagnosis and prognosis to the patients first, whereas $39.9 \%$ of the participants chose to disclose it to Family/Caregivers first. Details were shown in Table 3.

\section{Decision making and end-of-life issues}

Q13-Q24 was to describe participants' knowledge and attitudes on decision making and EOF issues. More than $50 \%$ of the participants had no idea of advanced directives (ADs) and do not resuscitate order (DNR). Only $7.2 \%$ of the participants approved that terminally ill cancer patients should received Cardiopulmonary Resuscitation (CPR) at the terminal stage. $58.7 \%$ of the participants though it was appropriate to discuss caseby-case according to the situations. $52.9 \%$ of them 
Table 2 General PC knowledge and attitudes

\begin{tabular}{|c|c|c|c|c|c|c|}
\hline \multicolumn{3}{|c|}{ Q1-Q5 items ${ }^{\mathrm{a}}$ (True, False, Unknown) } & \multicolumn{2}{|c|}{ Correct } & Number & Percent \\
\hline \multicolumn{7}{|c|}{$\begin{array}{l}\text { Q1. Palliative care should be provided for patients for whom no curative } \\
\text { treatments are available }\end{array}$} \\
\hline \multicolumn{3}{|l|}{$\begin{array}{l}\text { Q2. Palliative care should not be provided alongside } \\
\text { anti-cancer treatment }\end{array}$} & \multicolumn{2}{|l|}{$\mathrm{F}$} & 129 & 93.5 \\
\hline \multicolumn{3}{|l|}{ Q3. Different people have different ideas about PC } & \multicolumn{2}{|l|}{$\mathrm{T}$} & 124 & 89.9 \\
\hline \multicolumn{3}{|l|}{ Q4. Patients who receive PC must accept death } & \multicolumn{2}{|l|}{$\mathrm{F}$} & 89 & 64.5 \\
\hline \multicolumn{3}{|l|}{ Q5. Palliative care is the same as hospice care } & \multicolumn{2}{|l|}{$\mathrm{F}$} & 117 & 84.8 \\
\hline \multicolumn{3}{|l|}{ Participants who answered everything correctly } & & & 55 & 39.9 \\
\hline \multicolumn{7}{|l|}{$\begin{array}{l}\text { Q6. In your opinion, under what conditions should } \\
\text { cancer patients receive PC? }\end{array}$} \\
\hline \multicolumn{3}{|l|}{ A. When cancer is first diagnosed } & \multicolumn{2}{|l|}{-} & 45 & 32.6 \\
\hline \multicolumn{3}{|l|}{$\begin{array}{l}\text { B.When patients can no longer undergo surgery, radiotherapy, } \\
\text { chemotherapy and other anti-cancer therapy }\end{array}$} & \multicolumn{2}{|l|}{-} & 102 & 73.9 \\
\hline \multicolumn{3}{|l|}{ C. When patients' symptoms can no longer be controlled } & \multicolumn{2}{|l|}{-} & 77 & 55.8 \\
\hline \multicolumn{3}{|l|}{ D. When patients are mentally disabled } & \multicolumn{2}{|l|}{-} & 65 & 47.1 \\
\hline \multicolumn{3}{|l|}{ E. When patients proactively request PC } & \multicolumn{2}{|l|}{-} & 57 & 41.3 \\
\hline \multicolumn{3}{|l|}{ F. When the estimated survival length is less than 3 months } & \multicolumn{2}{|l|}{-} & 80 & 58.0 \\
\hline \multicolumn{3}{|l|}{ G. When the estimated survival length is less than 6 months } & \multicolumn{2}{|l|}{-} & 48 & 34.8 \\
\hline \multicolumn{3}{|l|}{ H. Others } & \multicolumn{2}{|l|}{-} & 13 & 9.4 \\
\hline \multirow[t]{2}{*}{ Q7-Q9 items ${ }^{c}$} & Yes & & \multicolumn{2}{|l|}{ No } & \multicolumn{2}{|l|}{ Depends } \\
\hline & N & $\%$ & $\mathrm{~N}$ & $\%$ & N & $\%$ \\
\hline $\begin{array}{l}\text { Q7.Would you recommend PC for cancer patients who } \\
\text { attend clinic for the first time? }\end{array}$ & 40 & 29.2 & 15 & 11.7 & 81 & 59.1 \\
\hline Q8.Do you believe that PC can improve patients' survival? & 75 & 54.3 & 8 & 5.8 & 55 & 39.3 \\
\hline $\begin{array}{l}\text { Q9.Do you believe that early PC integration can } \\
\text { improve patients' QOL? }\end{array}$ & 100 & 72.5 & 9 & 6.5 & 29 & 21.0 \\
\hline
\end{tabular}

${ }^{\mathrm{a}} \mathrm{Q} 1-\mathrm{Q} 5$, Respondents could respond with True, False, or Unknown. " $\mathrm{N}$ " represents the number of respondents who answered correctly

${ }^{\mathrm{b}} \mathrm{Q} 6$, Respondents could select more than one item in response. Thus, the percentages of Q6 add up to more than $100 \%$

'Q7-Q9, Respondents could select only one answer

would approve patients' wish when patients and family had conflicts on the decision making. If the patient was no longer competent and the family's wishes conflicted with those previous expressed by the patient, $38.4 \%$ of the participants would choose to support patients' wish. Details were in shown in Table 4. Only 20 (14.5\%) participants were reported to be familiarity with all five concepts listed in the Table 5. The greatest proportion $(77.5 \%)$ reported being familiar with euthanasia. Physician-assisted suicide was the most unfamiliar concept, known by only 34 participants $(24.6 \%)$.

\section{Discussion}

This is the first study to assess Chinese oncologists' knowledge, attitudes and practice towards PC and EOF issues. Our findings were some of the supplement of the previous studies conducted in Asian countries on physicians' attitudes.

Participants in this study had insufficient knowledge about PC. The insufficiency knowledge on PC may due to the lacking of formal PC education and training. Most worldwide physicians agree that current undergraduate and postgraduate programs do not provide sufficient education on PC [9]. In comparison to Western countries, PC education had not yet been institutionalized with respect to either the medical educational system or gaining the official status that other medical specialties hold, even oncology. PC has not been recognized as an important specialty in China yet. Although, more than $80 \%$ of Chinese interns in previous research felt that more education about PC should be included in the basic medical curriculum and clinical intern training [10]. In this survey, a large number of participants $(68.8 \%)$ had never received any formal education about PC. The lack of knowledge of PC among oncologists is one of the most common barriers to high quality PC services. More attention urgently needs to be paid on PC knowledge among the Oncologists in Mainland China.

Although a number of publications have recommended early access to PC for cancer patients. PC was 
Table 3 Disease information disclosure and breaking bad news

\begin{tabular}{lcc}
\hline Q10-Q12 $^{\prime}$ items $^{\text {a }}$ & Number & Percent \\
\hline Q10. Do you believe that you should inform patients of an unfavorable \\
prognosis? & 26 & 18.8 \\
Yes & 2 & 1.4 \\
No & 29 & 21.0 \\
It depends on patients' ${ }^{\prime}$ wishes & 20 & 14.5 \\
It depends on family/caregivers' wishes & 61 & 44.2 \\
It depends on situations & \\
Q11. Who should disclose information to patients? & \\
Doctor in charge & 102 & 73.9 \\
Family/Caregivers & 30 & 21.7 \\
Social volunteers & 3 & 2.2 \\
Others & 3 & 2.2 \\
Q12. Which person would you prefer to inform about the diagnosis and \\
prognosis? \\
Patients & 11 & 8.0 \\
Family/Caregivers & 55 & 39.9 \\
It depends on patients' wishes & 34 & 24.6 \\
It depends on family/caregivers wishes & 38 & 27.5 \\
\hline
\end{tabular}

${ }^{a} \mathrm{Q} 10-\mathrm{Q} 12$, Respondents could select only one answer

blt depends on the situation meant to decide on a case-by-case basis by considering the physical and psychological conditions, religion, and the cultural background of each individual

reported to be implemented late in the disease trajectory in previous studies [11]. The timing of referral to $\mathrm{PC}$ is a complex and dynamic process involving a wide range of dimensions [12]. Theoretically, oncologists' attitudes toward $\mathrm{PC}$ is one of the factors contributing to referrals time $[13,14]$. In our survey, only $29.2 \%$ of the participants would recommend $\mathrm{PC}$ for cancer patients when the patients attended the clinical first time. Although $72.5 \%$ believed that early PC integration could improve patients' QOL. This phenomenon indicated that further education regarding early PC integration should focus not only on patients but also on their doctor-in-charge.

Disease information disclosure, especially how to breaking bad news is one of the important issues in PC. According to the tort liability laws of China (Chapter VII liability for damages caused by medical treatment, Number 55), the medical staff shall explain to patients the situation regarding his illness and the measures for medical treatment. If it was not advisable to explain directly to patients, the explanation of the same should be served to their close family members which written consent should be obtained. Most physicians in Northern Europe and the US would usually reveal the diagnosis to the cancer patient. The way to deliver bad news in oncology is influenced by legal, ethical and cultural aspects [15]. A previous study on 60 oncology clinicians shown that only $40.5 \%$ of oncologists believed patients with terminal illness should be informed of the truth $[4,16]$. The situation was similar, in our survey, although most of the oncology clinicians $(73.9 \%)$ believed that the doctor-in-charge was the appropriate one to inform the patient of the diagnosis, Only 11 (8.0\%) clinicians believed that patients should be the first choice for information disclosure. Oncologists often found themselves in conflict with their medical teaching, cultural values, patient desires, family demands, or spiritual beliefs. For Chinese oncologists, disclosing diagnosis and prognosis to patients represented a big challenge, because they were confronted with a family-centered model of decision making [17]. Chinese oncologists had to face the dilemma of respecting patients and families' concerns about beneficence [2]. Whether family's points of view have some coincident with patients' standpoint need to be conducted by further researches focused on patients' attitudes.

ADs serves as a legal document that allows competent patients to give instructions regarding the health care they would like to receive during a time crisis when they will not be competent to make their own decisions. Although ADs have been widely advocated in Western countries, such as the USA, Canada, UK, the Netherlands, and Switzerland, only a few studies dealing with ADs in Asian countries have been reported [18-21]. Most of these studies focused on the attitudes of patients towards Ads. Thus, little is known concerning the attitudes of Asian physicians, especially in Mainland China, where there is no legalization of ADs [22, 23]. According to our survey, Chinese oncologists had insufficient knowledge about AD policy, much less knowledge than other Asian studies. But the ration was similar to a previous survey focused on medical staff's attitudes of ADs in Mainland China (26.8 \% vs $16.7 \%$ ). There exists a large gap in the degree of familiarity with ADs between Western and Eastern countries. The large gap also exists between Mainland China and other Asian countries.

The last part of the questionnaire gauges physicians' familiarity with five end-of-life issues: euthanasia, active euthanasia, passive euthanasia, physician-assisted suicide, and palliative sedation. Chinese oncologists were largely unfamiliar with most of the concepts presented. As for questions such as oncologists' attitudes and their intention to practice euthanasia were not included in the survey, because euthanasia and related end of life issues remains sensitive and controversial issue in Mainland China.

\section{Limitations}

The study had some limitations. First, the sample size of this survey was relatively small. The opinions expressed in this survey could not represent those of all Chinese oncologists. Second, the survey only evaluated oncologists' general 
Table 4 Decision making and end of life issues

\begin{tabular}{|c|c|c|c|c|c|c|}
\hline \multirow[t]{2}{*}{ Q13-Q15 items } & \multicolumn{2}{|c|}{ Yes } & \multicolumn{2}{|c|}{ No } & \multicolumn{2}{|c|}{ Not clearly } \\
\hline & N & $\%$ & N & $\%$ & N & $\%$ \\
\hline Q13.Do you know what advanced directives (ADs) is? & 37 & 26.8 & 69 & 50.0 & 32 & 23.2 \\
\hline Q14.Do you know what a do not resuscitate (DNR) order is? & 26 & 18.8 & 80 & 58.0 & 32 & 23.2 \\
\hline $\begin{array}{l}\text { Q15.Do you think you should follow a patient's wish when } \\
\text { he prefers to forgo life sustaining treatments? }\end{array}$ & 75 & 54.3 & 9 & 6.5 & 54 & 39.1 \\
\hline Q16-Q17 items & & & & & N & $\%$ \\
\hline \multicolumn{7}{|l|}{ Q16. Do you approve using CPR for terminally ill cancer patients? } \\
\hline Yes & & & & & 10 & 7.2 \\
\hline No & & & & & 47 & 34.1 \\
\hline It depends on the situation ${ }^{\mathrm{b}}$ & & & & & 81 & 58.7 \\
\hline \multicolumn{7}{|c|}{ Q17. 'What factors do you believe will affect a patient and family's decision? } \\
\hline A. Disease prognosis & & & & & 99 & 71.7 \\
\hline B. Symptom burden & & & & & 58 & 42.0 \\
\hline C. Other disease and comorbidities & & & & & 67 & 48.6 \\
\hline D. Religious beliefs & & & & & 75 & 54.3 \\
\hline E. Economic status & & & & & 88 & 63.8 \\
\hline F. The patient's own wishes/preferences & & & & & 82 & 59.4 \\
\hline G. Past experiences with death & & & & & 69 & 50.0 \\
\hline All selected & & & & & 32 & 23.2 \\
\hline
\end{tabular}

Q18-Q19

Q18. Should a conflict arise between the patient's wishes and the family's wishes in the decision-making process, who would you support?

Patients

Family

It depends on the situation ${ }^{\text {b }}$

Q19. If the patient is no longer competent and the family's wishes conflict with those previous expressed by the patient, who would you support?

Patients

Family

It depends on situation

${ }^{\mathrm{a}} \mathrm{Q} 17$ : Respondents could select more than one item in response. Thus, the percentages of Q6 add up to more than $100 \%{ }^{\mathrm{b}}$ It depends on the situation meant to decide on a case-by-case basis by considering the physical and psychological conditions, religion, and the cultural background of each individual

Table 5 Euthanasia and related issues

\begin{tabular}{|c|c|c|c|c|c|c|}
\hline \multirow[t]{2}{*}{ Q 20-24 } & \multicolumn{2}{|l|}{ Yes } & \multicolumn{2}{|l|}{ No } & \multicolumn{2}{|c|}{ Not clearly } \\
\hline & $\mathrm{N}$ & $\%$ & $\mathrm{~N}$ & $\%$ & $\mathrm{~N}$ & $\%$ \\
\hline Q20.Do you know what euthanasia is? & 107 & 77.5 & 4 & 2.9 & 27 & 19.6 \\
\hline Q21.Do you know what active euthanasia is? & 63 & 45.7 & 15 & 10.9 & 60 & 43.5 \\
\hline Q22.Do you know what passive euthanasia is? & 44 & 31.9 & 23 & 16.7 & 71 & 51.4 \\
\hline Q23.Do you know what physician-assisted suicide is? & 34 & 24.6 & 29 & 20.6 & 75 & 54.3 \\
\hline Q24.Do you know what palliative sedation is? & 44 & 31.9 & 27 & 19.6 & 47.5 & 48.6 \\
\hline Participants familiar with all concepts & 20 & 14.5 & & & & \\
\hline
\end{tabular}


knowledge and attitudes. This was due both to the short length of the survey and also to the intentionally superficial nature of the questions included. The questions did not delve deeply into how participants might think of certain concepts for two reasons. First, certain topics for example ADs, was still controversial and it was difficult to discuss the topic in detail in Mainland China. Second, many Chinese doctors may not have extensive knowledge of what $\mathrm{ADs}$ is, and asking more detailed questions without first establishing a clear definition would not be productive. To provide more information on specific issues, further education and surveys should be developed, and relevant factors should be analyzed for these issues.

\section{Conclusions}

This study provided a descriptive analysis of oncologists' knowledge, attitudes and practice toward PC and EOF issues in Mainland China. Chinese oncologists' knowledge toward $\mathrm{PC}$ and related issues were insufficient. To provide more information on specific issues, further surveys should be developed, and relevant factors should be analyzed for these issues. And further education program including knowledge and practical recommendations on PC urgently needs more attention in Mainland China.

\section{Abbreviation \\ PC: palliative care; EOF: end of life; Q1-Q24: Question 1-Question 24; FUSCC: Fudan University Shanghai Cancer Center; QOL: quality of life: ADs: Advanced directives; DNR: do not resuscitate order; TCM: Traditional Chinese Medicine; TCH: Tertiary Comprehensive Hospital; SCH: Secondary Comprehensive Hospital; PCH: Primary community hospital.}

\section{Competing interest}

The authors have no conflicts of interest to declare. This study has no financial relationship with any organization that sponsored the research and authorship. The corresponding author has full control of all primary data and will allow the journal to review the data if requested.

\section{Authors' contributions}

XLG had primay responsibility for developing study protocol, enrollingment of the participants and drafting the manuscripting, performing the statistical analyses and revising the manuscript. WCC supervised the study. Both authors read, approved and contributed to the final paper.

\section{Acknowledgments}

We gratefully acknowledge palliative care specialists in Department of Integrated Therapy, Fudan University Shanghai Cancer Center for participating in the study. We aslo gratefully acknowledge all the oncologists participated in the survey.

Received: 21 September 2015 Accepted: 12 May 2016

Published online: 18 May 2016

\section{References}

1. Wright M, Wood J, Lynch T, Clark D. Mapping levels of palliative care development: A global view. J Pain Symptom Management. 2008;355:469-85.

2. Wang SY, Chen $\mathrm{CH}$, Chen YS, Huang HL. The attitude toward truth telling of cancer in Taiwan. J Psychosom Res. 2004;57(1):53-8.

3. Liu L, Yuan C. Construction of Palliative Care Training Contents in China: A Delphi Study. Cancer Nurs. 2009:32(6):446-55.

4. Wang XS, Di LJ, Reyes-Gibby CC, Guo H, Liu SJ, Cleeland CS. End-of-life care in urban areas of China: A survey of 60 oncology clinicians. J Pain Symptom Management. 2004;27(2):125-32.
5. Yamamoto R, Kizawa Y, Nakazawa Y, Morita T. The Palliative Care Knowledge Questionnaire for PEACE: Reliability and Validity of an Instrument To Measure Palliative Care Knowledge among Physicians. J Palliat Med. 2013;16(11):1423-8.

6. Hong CY, Ng GN, Poulose J, Lin NJ, Goh CR. Attitude of doctors in an Asian Oncology centre towards referral to Palliative Care. J Palliat Med. 2011;27(2):157-63.

7. Sittisombut S, Maxwell C, Love EJ, Sitthi-Amorn C. Physicians' attitudes and practices regarding advanced end-of-life care planning for terminally ill patients at Chiang Mai University Hospital, Thailand. Nurs Health Sci. 2009;11(1):23-8.

8. Bradley EH, Cramer LD, Bogardus Jr ST, Kasl SV, Johnson-Hurzeler R, Horwitz SM. Physicians' Ratings of Their Knowledge, Attitudes, and End-of-life-care Practices. Acad Med. 2002;77(4):305-11.

9. Nakazawa Y, Miyashita M, Morita T, Umeda M, Oyagi Y, Ogasawara T. The palliative care knowledge test: reliability and validity of an instrument to measure palliative care knowledge among health professionals. Palliat Med. 2009;23(8):754-66.

10. Jiang X, Liao Z, Hao J, Guo Y, Zhou Y, Ning L, et al. Palliative Care Education in China: Insight Into One Medical University. J Pain Symptom Management. 2011;41(4):796-800.

11. Christakis NA, Iwashyna TJ. Attitude and self-reported practice regarding prognostication in a national sample of internists. Arch Intern Med. 1998; 158(21):2389-95.

12. Greer JA, Jackson VA, Meier DE, Temel JS. Early integration of palliative care services with standard oncology care for patients with advanced cancer. Caa Cancer J Clin. 2013;63(5):349-63.

13. Snyder S, Hazelett S, Allen K, Radwany S. Physician Knowledge, Attitude, and Experience With Advance Care Planning, Palliative Care, and Hospice: Results of a Primary Care Survey. Am J Hosp Palliat Care. 2013;30(5):419-24.

14. Schenker Y, Crowley-Matoka M, Dohan D, Rabow MW, Smith CB, White DB, et al. Oncologist factors that influence referrals to subspecialty palliative care clinics. J Oncol Pract. 2014;10(2):e37-44.

15. Ong KJ, Back MF, Lu JJ, Shakespeare TS, Wynne CJ. Cultural attitudes to cancer management in traditional South-East Asian patients. Australas Radiol. 2002:46(4):370-4

16. Jiang Y, Li J-Y, Liu C, Huang MJ, Zhou L, Li M, et al. Different attitudes of oncology clinicians toward truth telling of different stages of cancer. Supportive Care Cancer. 2006;14(11):1119-25.

17. Back MF, Huak CY. Family centred decision making and non-disclosure of diagnosis in a South East Asian oncology practice. Psycho-Oncol. 2005; 14(12):1052-9.

18. Miura Y, Asai A, Nagata S, Ohnishi M, Shimbo T, Hosoya T, et al. Dialysis patients' preferences regarding cardiopulmonary resuscitation and withdrawal of dialysis in Japan. Am J Kidney Dis. 2001;37(6):1216-22.

19. Sittisombut S, Love EJ, Sitthi-Amorn C. Attitudes toward advance directives and the impact of prognostic information on the preference for cardiopulmonary resuscitation in medical inpatients in Chiang Mai University Hospital, Thailand. Nurs Health Sci. 2005;7(4):243-50.

20. Low JA, Ng WC, Yap KB, Chan KM. End-of-life issues: Preferences and choices of a group of elderly Chinese subjects attending a day care centre in Singapore. Ann Acad Med Singapore. 2000;29(1):50-6.

21. Akabayashi A, Slingsby BT, Kai I. Perspectives on advance directives in Japanese society: A population-based questionnaire survey. BMC Med Ethics. 2003;31(4):E5-5.

22. Tee KH, Seet LT, Tan WC, Choo HW. Advance directive: A study on the knowledge and attitudes among general practitioners in Singapore. Smj. 1997:38(4):145-8.

23. Asai A, Miura Y, Tanabe N, Kurihara M, Fukuhara S. Advance directives and other medical decisions concerning the end of life in cancer patients in Japan. Euro J Cancer. 1998;34(10):1582-6. 